\title{
RETRACTED ARTICLE: Serum Zinc and Adiponectin Levels in Patients with Polycystic Ovary Syndrome, Adjusted for Anthropometric, Biochemical, Dietary Intake, and Physical Activity Measures
}

\author{
Sahar Mazloomi ${ }^{1,2}$ • Narges Alizadeh ${ }^{3}$ - Majid Aminzare ${ }^{4}$. \\ Soudabeh Niroomand $^{5}$ - Seyedeh Neda Mousavi ${ }^{1}$
}

Received: 23 October 2016 / Accepted: 24 January 2017 /Published online: 3 February 2017

(C) Springer Science+Business Media New York 2017

The authors retract this article. The authors have declared that the underlying data may not have been gathered and assessed correctly. The article also has significant statistical inconsistencies. The data reported in this article are therefore unreliable. Not all authors have responded to correspondence from the editor about this retraction.

The online version of this article contains the full text of the retracted article as electronic supplementary material.

Electronic supplementary material The online version of this article (https://doi.org/10.1007/s12011-017-0951-0) contains supplementary material, which is available to authorized users.

Seyedeh Neda Mousavi

neda.mousavi@zums.ac.ir

1 Department of Biochemistry and Nutrition, School of Medicine, Zanjan University of Medical Sciences, Zanjan, Iran

2 Department of Internal Medicine, Metabolic Disease Research Center, Vali-e-Asr Hospital, Zanjan University of Medical Sciences, Zanjan, Iran

3 Department of Obstetrics and Gynecology, Vali-e-Asr Hospital, Zanjan University of Medical Sciences, Zanjan, Iran

4 Department of Food Safety and Hygiene, Faculty of Health, Zanjan University of Medical Sciences, Zanjan, Iran

5 Department of Midwifery, School of Nursing and Midwifery, Zanjan University of Medical Sciences, Zanjan, Iran 